\title{
The Uniqueness Theorem of the Solution for a Class of Differential Systems with Coupled Integral Boundary Conditions
}

\author{
Shuman Meng ${ }^{1}$ and Yujun Cui ${ }^{1,2}$ \\ ${ }^{1}$ Department of Applied Mathematics, Shandong University of Science and Technology, Qingdao 266590, China \\ ${ }^{2}$ State Key Laboratory of Mining Disaster Prevention and Control Co-Founded by Shandong Province and the Ministry of \\ Science and Technology, Shandong University of Science and Technology, Qingdao 266590, China
}

Correspondence should be addressed to Yujun Cui; cyj720201@163.com

Received 16 April 2018; Accepted 3 July 2018; Published 11 July 2018

Academic Editor: Jorge E. Macias-Diaz

Copyright (C) 2018 Shuman Meng and Yujun Cui. This is an open access article distributed under the Creative Commons Attribution License, which permits unrestricted use, distribution, and reproduction in any medium, provided the original work is properly cited.

We discuss the uniqueness of the solution to a class of differential systems with coupled integral boundary conditions under a Lipschitz condition. Our main method is the linear operator theory and the solvability for a system of inequalities. Finally, an example is given to demonstrate the validity of our main results.

\section{Introduction}

In this paper, we study the following differential system with coupled integral boundary conditions:

$$
\begin{aligned}
-u^{\prime \prime}(t) & =f(t, u(t), v(t)), \quad t \in(0,1), \\
-v^{\prime \prime}(t) & =g(t, u(t), v(t)), \quad t \in(0,1), \\
u(0) & =v(0)=0, \\
u(1) & =\alpha[v], \\
v(1) & =\beta[u],
\end{aligned}
$$

where $\alpha[u], \beta[u]$ are bounded linear functionals on $C[0,1]$ given by

$$
\begin{aligned}
& \alpha[u]=\int_{0}^{1} u(t) d A(t), \\
& \beta[u]=\int_{0}^{1} u(t) d B(t),
\end{aligned}
$$

involving Riemann-Stieltjes integrals defined via positive Stieltjes measures of $A, B$.

Differential systems with coupled boundary conditions have some applications in various fields of sciences and engineering, for example, the heat equation [1], reactiondiffusion phenomena [2], and interaction problems [3]. The existence of solutions for differential system with coupled boundary conditions has received a growing attention in the literature; for details, see [4-21]. For example, Asif and Khan in [4] obtained the existence of positive solution for singular sublinear system with coupled four-point boundary value conditions by using the Guo-Krasnosel'skii fixed point theorem. In [5], Cui and Sun discuss the existence of positive solutions of singular superlinear coupled integral boundary value problems by constructing a special cone and using fixed point index theory. In [7], Cui and Zou proved the existence of extremal solutions of coupled integral boundary value problems by monotone iterative method. In [10], Infante, Minhós, and Pietramala presented a general theory for existence of positive solutions for coupled systems by use of fixed point index theory.

The question of existence and uniqueness of solution of differential equations and differential systems is an ageold problem and it has a great importance, as much in theory as in applications. This problem has been investigated by use of a variety of nonlinear analyses such as fixed point theorem for mixed monotone operator $[7,15,22-$ 25], maximal principle [6], Banach's contraction mapping principle [26-29], and the linear operator theory $[27,30,31]$. 
For example, the authors [31] introduced a Banach space using the positive eigenfunction of linear operator related to differential system (1). They established the uniqueness results for differential system (1) under a Lipschitz condition. It should be noted that the Lipschitz constant is related to the spectral radius corresponding to the related linear operators. The obtained results are optimal from the viewpoint of theory. However, it is very difficult to determine the spectral radius for differential system (1) with general functions $A(t), B(t)$.

Motivated by the above works, we investigate the uniqueness of solutions for differential system (1) by using a system of inequalities and the linear operator theory. The main features of this paper are as follows: (1) The main results are mostly implemented to the uniqueness result for coupled boundary value problems. (2) An easy criterion to determine the uniqueness result is obtained by using a system of inequalities. (3) An example shows that the main result provides the same results with weaker conditions.

Throughout the paper, we assume that the following condition hold:

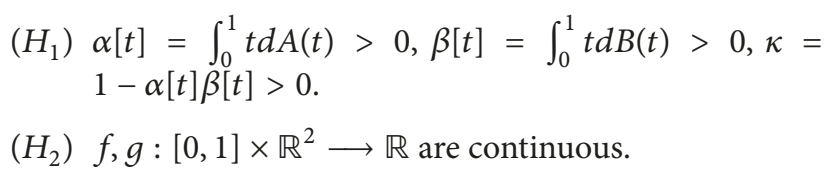

\section{Preliminaries}

Let $C[0,1]$ be the Banach space with the maximal norm given by $\|x\|=\max _{t \in[0,1]}|x(t)|$. Let $E=C[0,1] \times C[0,1],\|(x, y)\|_{E}=$ $\max \{\|x\|,\|y\|\}$. Then $\left(E,\|(\cdot, \cdot)\|_{E}\right)$ is a Banach space.

Lemma 1 (see [5]). Let $u, v \in C[0,1]$, then the system of BVPs

$$
\begin{aligned}
-u^{\prime \prime}(t) & =x(t), \\
-v^{\prime \prime}(t) & =y(t), \\
t \in[0,1], & \\
u(0) & =v(0)=0, \\
u(1) & =\alpha[v], \\
v(1) & =\beta[u]
\end{aligned}
$$

has integral representation

$$
\begin{aligned}
& u(t)=\int_{0}^{1} G_{1}(t, s) x(s) d s+\int_{0}^{1} H_{1}(t, s) y(s) d s, \\
& v(t)=\int_{0}^{1} G_{2}(t, s) y(s) d s+\int_{0}^{1} H_{2}(t, s) x(s) d s,
\end{aligned}
$$

where

$$
\begin{aligned}
& G_{1}(t, s)=\frac{\alpha[t] t}{\kappa} \int_{0}^{1} k(s, \tau) d B(\tau)+k(t, s), \\
& H_{1}(t, s)=\frac{t}{\kappa} \int_{0}^{1} k(s, \tau) d A(\tau),
\end{aligned}
$$

$$
\begin{aligned}
G_{2}(t, s) & =\frac{\beta[t] t}{\kappa} \int_{0}^{1} k(s, \tau) d A(\tau)+k(t, s), \\
H_{2}(t, s) & =\frac{t}{\kappa} \int_{0}^{1} k(s, \tau) d B(\tau), \\
k(t, s) & = \begin{cases}t(1-s), & 0 \leq t \leq s \leq 1, \\
s(1-t), & 0 \leq s \leq t \leq 1 .\end{cases}
\end{aligned}
$$

Lemma 2 (see [5]). The functions $k(t, s), G_{i}(t, s), H_{i}(t, s)(i=$ $1,2)$ satisfy the following properties:

$$
\begin{aligned}
G_{i}(t, s) & \leq \rho t, \\
H_{i}(t, s) & \leq \rho t, \\
& \forall t, s \in[0,1], i=1,2, \\
& 0<k(t, s) \leq s(1-s), \quad \forall t, s \in(0,1),
\end{aligned}
$$

where

$$
\begin{aligned}
\rho= & \max \left\{\frac{\alpha[t]}{\kappa} \beta[1]+1, \frac{\beta[t]}{\kappa} \alpha[1]\right. \\
& \left.+1, \frac{1}{\kappa} \beta[1], \frac{1}{\kappa} \alpha[1]\right\} .
\end{aligned}
$$

With the help of Lemma 1, BVP (1) can be viewed as a fixed point in $E$ for the completely continuous operator

$$
S(u, v)=\left(S_{1}(u, v), S_{2}(u, v)\right), \quad(u, v) \in E,
$$

where $S_{1}, S_{2}: E \rightarrow C[0,1]$ are defined by

$$
\begin{aligned}
S_{1}(u, v)(t)= & \int_{0}^{1} G_{1}(t, s) f(s, u(s), v(s)) d s \\
& +\int_{0}^{1} H_{1}(t, s) g(s, u(s), v(s)) d s, \\
S_{2}(u, v)(t)= & \int_{0}^{1} G_{2}(t, s) g(s, u(s), v(s)) d s \\
& +\int_{0}^{1} H_{2}(t, s) f(s, u(s), v(s)) d s .
\end{aligned}
$$

In order to prove our main result, the following criterion for solving system of inequalities is needed.

Lemma 3. Let $a, b, c, d \in[0,+\infty)$ with $a<1, d<1$. Then the inequality system

$$
\begin{aligned}
& a+b \mu \leq \lambda, \\
& c+d \mu \leq \lambda \mu
\end{aligned}
$$

has a solution $(\lambda, \mu)$ with $\lambda \in(0,1), \mu>0$ if and only if $a, b, c, d$ satisfy

$$
(1-d)(1-a)>b c
$$


Proof.

Necessity. The proof is obviously true for the case: $b c=0$. So we consider the remaining case $b c \neq 0$. From the first inequality in (11), we get

$$
\mu \leq \frac{\lambda-a}{b} .
$$

Substituting it into the second inequality in (11), we have

$$
c \leq(\lambda-d) \mu \leq(\lambda-d) \frac{\lambda-a}{b} .
$$

Thus,

$$
(1-d)(1-a)>(\lambda-d)(\lambda-a) \geq b c
$$

Sufficiency. For the case $b c=0$, we can take $\lambda=\max \{(d+$ $1) / 2,(d+1) / 2\}$. So we consider the last case $b c \neq 0$. Let

$$
\varphi(x)=(x-d)(x-a)-b c, \quad x \in \mathbb{R} .
$$

From the derivative of $\varphi(x)$, we conclude that $\varphi(x)$ is increasing on $[(a+d) / 2,1]$. This together with the locally sign-preserving property of $\varphi(x)$ implies that there exists $\lambda \epsilon$ $[(a+d) / 2,1)$ such that

$$
(\lambda-d)(\lambda-a) \geq b c
$$

The above inequality can be rewritten as

$$
\frac{c}{\lambda-d} \leq \frac{\lambda-a}{b}
$$

Hence (11) holds for $\mu \in[c /(\lambda-d),(\lambda-a) / b]$.

\section{Main Result}

For notational convenience, let

$$
\begin{aligned}
& a_{11}=\frac{\alpha[t] \beta[\phi]}{\kappa}+\frac{1}{6}, \\
& a_{12}=\frac{\alpha[\phi]}{\kappa}, \\
& a_{21}=\frac{\alpha[\phi] \beta[t]}{\kappa}+\frac{1}{6}, \\
& a_{22}=\frac{\beta[\phi]}{\kappa},
\end{aligned}
$$

where

$$
\phi(t)=\frac{t(1-t)(1+t)}{6} .
$$

Take $\varphi(t)=t$. By $(7)$, we get

$$
\begin{aligned}
\int_{0}^{1} G_{1}(t, s) \varphi(s) d s \\
=\frac{\alpha[t] t}{\kappa} \int_{0}^{1} \int_{0}^{1} k(s, \tau) d B(\tau) s d s+\int_{0}^{1} k(t, s) s d s \\
=\frac{\alpha[t] t}{\kappa} \int_{0}^{1} \int_{0}^{1} k(s, \tau) s d s d B(\tau)+\frac{t(1-t)(1+t)}{6} \\
=\frac{\alpha[t] t}{\kappa} \int_{0}^{1} \frac{\tau(1-\tau)(1-\tau)}{6} d B(\tau) \\
\quad+\frac{t(1-t)(1+t)}{6} \leq\left(\frac{\alpha[t] \beta[\phi]}{\kappa}+\frac{1}{6}\right) \cdot t
\end{aligned}
$$$$
=a_{11} t
$$$$
\int_{0}^{1} H_{1}(t, s) \varphi(s) d s=\frac{t}{\kappa} \int_{0}^{1} \int_{0}^{1} k(s, \tau) d A(\tau) s d s
$$$$
=\frac{t}{\kappa} \int_{0}^{1} \int_{0}^{1} k(s, \tau) s d s d A(\tau)
$$$$
=\frac{t}{\kappa} \int_{0}^{1} \frac{\tau(1-\tau)(1-\tau)}{6} d A(\tau)=\frac{\alpha[\phi]}{\kappa} \cdot t=a_{12} t,
$$

$$
\begin{aligned}
\int_{0}^{1} G_{2}(t, s) \varphi(s) d s & \\
= & \frac{\beta[t] \alpha[\varphi] t}{\kappa} \int_{0}^{1} \int_{0}^{1} k(s, \tau) d A(\tau) s d s \\
& +k(t, s) s d s \\
= & \frac{\beta[t] t}{\kappa} \int_{0}^{1} \frac{\tau(1-\tau)(1-\tau)}{6} d A(\tau) \\
& +\frac{t(1-t)(1+t)}{6} \leq\left(\frac{\alpha[\phi] \beta[t]}{\kappa}+\frac{1}{6}\right) \cdot t \\
\int_{0}^{1} & a_{21} t, \\
= & \frac{t}{\kappa} \int_{0}^{1} \int_{0}^{1} k(s, \tau) s d s d B(\tau) \\
= & \frac{t}{\kappa} \int_{0}^{1} \frac{\tau(1-\tau)(1-\tau)}{6} d B(\tau)=\frac{\beta[\phi]}{\kappa} \cdot t=a_{22} t .
\end{aligned}
$$

By use of (21), (22), (23), and (24), we present the main result of this paper.

Theorem 4. Suppose that there exist four nonnegative constants $a_{1}, b_{1}, c_{1}, d_{1}$ such that the following conditions hold:

$$
\begin{aligned}
& \quad\left|f\left(t, u_{1}, v_{1}\right)-f\left(t, u_{2}, v_{2}\right)\right| \\
& \leq a_{1}\left|u_{1}-u_{2}\right|+b_{1}\left|v_{1}-v_{2}\right|, \\
& \quad t \in[0,1], u_{1}, u_{2}, v_{1}, v_{2} \in \mathbb{R},
\end{aligned}
$$




$$
\begin{aligned}
& \left|g\left(t, u_{1}, v_{1}\right)-g\left(t, u_{2}, v_{2}\right)\right| \\
& \leq c_{1}\left|u_{1}-u_{2}\right|+d_{1}\left|v_{1}-v_{2}\right|, \\
& t \in[0,1], u_{1}, u_{2}, v_{1}, v_{2} \in \mathbb{R}, \\
& \left(1-a_{11} a_{1}-a_{12} c_{1}\right)\left(1-a_{21} d_{1}-a_{22} b_{1}\right) \\
& >\left(a_{11} b_{1}+a_{12} d_{1}\right)\left(a_{21} c_{1}+a_{22} a_{1}\right), \\
& a_{11} a_{1}+a_{12} c_{1}<1, \\
& a_{21} d_{1}+a_{22} b_{1}<1 \text {. }
\end{aligned}
$$

\section{Then differential system (1) has a unique solution in $E$.}

Proof. We divide the proof into several main steps to show that the operator $S$ has a unique point in $E$ under the conditions of Theorem 4 .

Step 1. It follows from (25), (26), and Lemma 3 that there exist $\lambda \in(0,1), \mu>0$ such that

$$
\begin{aligned}
& \left(a_{11} a_{1}+a_{12} c_{1}\right)+\left(a_{11} b_{1}+a_{12} d_{1}\right) \mu \leq \lambda, \\
& \left(a_{21} c_{1}+a_{22} a_{1}\right)+\left(a_{21} d_{1}+a_{22} b_{1}\right) \mu \leq \lambda \mu .
\end{aligned}
$$

Let us introduce a linear operator $T$ on $E$ as

$$
T(u, v)=\left(T_{1}(u, v), T_{2}(u, v)\right)
$$

where $T_{1}, T_{2}: E \longrightarrow C[0,1]$ is given by

$$
\begin{aligned}
T_{1}(u, v)(t)= & \int_{0}^{1} G_{1}(t, s)\left(a_{1} u(s)+b_{1} v(s)\right) d s \\
& +\int_{0}^{1} H_{1}(t, s)\left(c_{1} u(s)+d_{1} v(s)\right) d s, \\
T_{2}(u, v)(t)= & \int_{0}^{1} G_{2}(t, s)\left(c_{1} u(s)+d_{1} v(s)\right) d s \\
& +\int_{0}^{1} H_{2}(t, s)\left(a_{1} u(s)+b_{1} v(s)\right) d s .
\end{aligned}
$$

Take $\psi(t)=\mu t$. Now, (21)-(24) and (27) show that

$$
\begin{aligned}
T_{1}(\varphi, \psi)(t)= & \int_{0}^{1} G_{1}(t, s)\left(a_{1} \varphi(s)+b_{1} \psi(s)\right) d s \\
& +\int_{0}^{1} H_{1}(t, s)\left(c_{1} \varphi(s)+d_{1} \psi(s)\right) d s \\
\leq & \left(a_{1} a_{11}+a_{12} c_{1}\right) \varphi(t) \\
& +\left(a_{11} b_{1}+a_{12} d_{1}\right) \psi(t) \leq \lambda \varphi(t),
\end{aligned}
$$

$$
\begin{aligned}
T_{2}(\varphi, \psi)(t)= & \int_{0}^{1} G_{2}(t, s)\left(c_{1} \varphi(s)+d_{1} \psi(s)\right) d s \\
& +\int_{0}^{1} H_{2}(t, s)\left(a_{1} \varphi(s)+b_{1} \psi(s)\right) d s \\
\leq & \left(c_{1} a_{21}+a_{22} c_{1}\right) \varphi(t) \\
& +\left(a_{21} d_{1}+a_{22} b_{1}\right) \psi(t) \leq \lambda \psi(t),
\end{aligned}
$$

i.e.,

$$
T(\varphi, \psi)(t) \leq \lambda(\varphi(t), \psi(t)) .
$$

Then for $p \in \mathbb{N}$, by induction, we obtain

$$
T^{p}(\varphi, \psi)(t) \leq \lambda^{p}(\varphi(t), \psi(t)) .
$$

Step 2. For all $(u, v) \in E$ with $u(t) \geq 0$ and $v(t) \geq 0$, there exists $M=M(u, v) \in(0,+\infty)$ such that

$$
T(u, v)(t) \leq M \cdot(\varphi(t), \psi(t)), \quad t \in[0,1] .
$$

Indeed, by Lemma 2, we have

$$
\begin{aligned}
T_{1}(u, v)(t) & \leq \rho\left(\left(a_{1}+c_{1}\right)\|u\|+\left(b_{1}+d_{1}\right)\|v\|\right) t \\
& =\rho\left(\left(a_{1}+c_{1}\right)\|u\|+\left(b_{1}+d_{1}\right)\|v\|\right) \varphi(t) \\
T_{2}(u, v)(t) & \leq \rho\left(\left(a_{1}+c_{1}\right)\|u\|+\left(b_{1}+d_{1}\right)\|v\|\right) t \\
& =\frac{\rho\left(\left(a_{1}+c_{1}\right)\|u\|+\left(b_{1}+d_{1}\right)\|v\|\right)}{\mu} \psi(t)
\end{aligned}
$$

So, we can take $M=\rho \max \{1,1 / \mu\}\left(\left(a_{1}+c_{1}\right)\|u\|+\left(b_{1}+d_{1}\right)\|v\|\right)$ such that (34) holds.

Step 3. For any given $\left(u_{0}, v_{0}\right) \in E, n=1,2, \ldots$, let $\left(u_{n}, v_{n}\right)=$ $S\left(u_{n-1}, v_{n-1}\right)$. By Step 2, there exists $M>0$ such that

$$
\begin{aligned}
& T\left(\left|u_{1}(t)-u_{0}(t)\right|,\left|v_{1}(t)-v_{0}(t)\right|\right) \\
& \quad \leq M \cdot(\varphi(t), \psi(t)), \quad t \in[0,1] .
\end{aligned}
$$

Notice for $p \in \mathbb{N}$ that

$$
\begin{aligned}
& \left|u_{n+p+1}(t)-u_{n+p}(t)\right|=\mid S_{1}\left(u_{n+p}, v_{n+p}\right)(t) \\
& -S_{1}\left(u_{n+p-1}, v_{n+p-1}\right)(t) \mid \leq \int_{0}^{1} G_{1}(t, s) \\
& \cdot \mid f\left(s, u_{n+p}(s), v_{n+p}(s)\right) \\
& -f\left(s, u_{n+p-1}(s), v_{n+p-1}(s)\right) \mid d s \\
& +\int_{0}^{1} H_{1}(t, s) \mid g\left(s, u_{n+p}(s), v_{n+p}(s)\right) \\
& -g\left(s, u_{n+p-1}(s), v_{n+p-1}(s)\right) \mid d s \\
& \leq \int_{0}^{1} G_{1}(t, s)\left(a_{1}\left|u_{n+p}(t)-u_{n+p-1}(t)\right|\right. \\
& \left.+b_{1}\left|v_{n+p}(t)-v_{n+p-1}(t)\right|\right) d s+\int_{0}^{1} H_{1}(t, s) \\
& \cdot\left(c_{1}\left|u_{n+p}(t)-u_{n+p-1}(t)\right|\right.
\end{aligned}
$$




$$
\begin{gathered}
\left.\quad+d_{1}\left|v_{n+p}(t)-v_{n+p-1}(t)\right|\right) d s=T_{1}\left(\mid u_{n+p}(t)\right. \\
\left.-u_{n+p-1}(t)|,| v_{n+p}(t)-v_{n+p-1}(t) \mid\right)(t) \leq \cdots \\
\leq T_{1}^{n+p}\left(\left|u_{1}-u_{0}\right|,\left|v_{1}-v_{0}\right|\right)(t), \\
\left|v_{n+p+1}(t)-v_{n+p}(t)\right| \leq T_{2}^{n+p}\left(\left|u_{1}-u_{0}\right|,\left|v_{1}-v_{0}\right|\right)(t) .
\end{gathered}
$$

Thus, by (33) and (36), we obtain that

$$
\begin{aligned}
& \left(\left|u_{n+p+1}(t)-u_{n+p}(t)\right|,\left|v_{n+p+1}(t)-v_{n+p}(t)\right|\right) \\
& \quad \leq T^{n+p}\left(\left|u_{1}(t)-u_{0}(t)\right|,\left|v_{1}(t)-v_{0}(t)\right|\right) \\
& \quad \leq M T^{n+p-1}(\varphi, \psi)(t) \leq M \lambda^{n+p-1}(\varphi(t), \psi(t)) .
\end{aligned}
$$

Thus for $n, m \in \mathbb{N}$, we conclude that

$$
\begin{aligned}
\left|u_{n+m}(t)-u_{n}(t)\right| \leq & \left|u_{n+m}(t)-u_{n+m-1}(t)\right|+\cdots \\
& +\left|u_{n+1}(t)-u_{n}(t)\right| \\
\leq & M\left(\lambda^{n+m-2}+\cdots+\lambda^{n-1}\right) \varphi(t) \\
= & M \frac{\lambda^{n-1}-\lambda^{n+m-1}}{1-\lambda} \varphi(t), \\
\left|v_{n+m}(t)-v_{n}(t)\right| \leq & M \frac{\lambda^{n-1}-\lambda^{n+m-1}}{1-\lambda} \psi(t) .
\end{aligned}
$$

The above two inequalities ensure that $\left\{\left(u_{n}, v_{n}\right)\right\}$ is a Cauchy sequence in $E$. Since $E$ is complete, there exists $\left(u^{*}, v^{*}\right) \in E$ such that $\lim _{n \rightarrow \infty}\left(u_{n}, v_{n}\right)=\left(u^{*}, v^{*}\right)$. Therefore, $\left(u^{*}, v^{*}\right)$ is a fixed point of $S$ that follows from the continuity of operator $S$.

Step 4. We show that $S$ has a unique fixed point. Suppose there exist two elements $\left(u^{*}, v^{*}\right),\left(u_{*}, v_{*}\right)$ with $S\left(u^{*}, v^{*}\right)=\left(u^{*}, v^{*}\right)$ and $S\left(u_{*}, v_{*}\right)=\left(u_{*}, v_{*}\right)$. By Step 2 , there exists $M>0$ such that

$$
\begin{gathered}
T\left(\left|u^{*}(t)-u_{*}(t)\right|,\left|v^{*}(t)-v_{*}(t)\right|\right) \\
\quad \leq M(\varphi(t), \psi(t)), \quad t \in[0,1] .
\end{gathered}
$$

Applying the method used in Step 3 again, for $p \in \mathbb{N}$, we get

$$
\begin{aligned}
& \left|u^{*}(t)-u_{*}(t)\right| \leq M \frac{\lambda^{p}}{1-\lambda} \varphi(t), \\
& \left|v^{*}(t)-v_{*}(t)\right| \leq M \frac{\lambda^{p}}{1-\lambda} \psi(t) .
\end{aligned}
$$

Hence we get the desired results. theory.
Example 5. Consider the differential system

$$
\begin{aligned}
-x^{\prime \prime}(t) & =\sin x(t)+\ln \left(1+y^{2}(t)\right)+h_{1}(t), \\
-y^{\prime \prime}(t) & =2 \arctan x(t)+\sqrt{1+y^{2}(t)}+h_{2}(t), \\
t \in(0,1), 1), & t \in(0, \\
x(0) & =y(0)=0, \\
x(1) & =\int_{0}^{1} y(t) d t, \\
y(1) & =2 \int_{0}^{1} x(t) d t,
\end{aligned}
$$

where $h_{1}, h_{2} \in C[0,1]$. We have

$$
\begin{aligned}
A(t) & =t, \\
B(t) & =2 t, \\
\alpha[t] & =\frac{1}{2}, \\
\beta[t] & =1, \\
\kappa & =\frac{1}{2}, \\
\alpha[\phi] & =\frac{1}{24}, \\
\beta[\phi] & =\frac{1}{12}, \\
a_{11} & =\frac{\alpha[t] \beta[\phi]}{\kappa}+\frac{1}{6}=\frac{1}{4}, \\
a_{12} & =\frac{\alpha[\phi]}{\kappa}=\frac{1}{12}, \\
a_{21} & =\frac{\alpha[\phi] \beta[t]}{\kappa}+\frac{1}{6}=\frac{1}{4}, \\
a_{22} & =\frac{\beta[\phi]}{\kappa}=\frac{1}{6} .
\end{aligned}
$$

Let

$$
\begin{aligned}
& f(t, x, y)=\sin x+\ln \left(1+y^{2}\right)+h_{1}(t), \\
& g(t, x, y)=2 \arctan x+\sqrt{1+y^{2}}+h_{2}(t),
\end{aligned}
$$

then

$$
\begin{aligned}
& \left|f\left(t, u_{1}, v_{1}\right)-f\left(t, u_{2}, v_{2}\right)\right| \leq\left|u_{1}-u_{2}\right|+\left|v_{1}-v_{2}\right|, \\
& \left|g\left(t, u_{1}, v_{1}\right)-g\left(t, u_{2}, v_{2}\right)\right| \leq 2\left|u_{1}-u_{2}\right|+\left|v_{1}-v_{2}\right|,
\end{aligned}
$$

where $t \in[0,1], u_{1}, u_{2}, v_{1}, v_{2} \in \mathbb{R}$. Hence, there exists a solution $(\lambda, \mu)=(11 / 12,3 / 2)$ of the following inequality system:

$$
\left(a_{11} a_{1}+a_{12} c_{1}\right)+\left(a_{11} b_{1}+a_{12} d_{1}\right) \mu=\frac{5}{12}+\frac{1}{3} \mu \leq \lambda,
$$




$$
\left(a_{21} c_{1}+a_{22} a_{1}\right)+\left(a_{21} d_{1}+a_{22} b_{1}\right) \mu=\frac{2}{3}+\frac{5}{12} \mu \leq \lambda \mu .
$$

Therefore, according to Theorem 4, the problem (42) has a unique solution.

When the nonlinearity of differential equation and differential system satisfies Lipschitz condition, the usual method to obtain the uniqueness is the well-known Banach's contraction principle. For this purpose, we should add some restriction on the Lipschitz constants to guarantee the norm of a linear operator related to differential equation and differential system less than 1 . Next, we discuss the estimate of the norm of a linear operator related to differential system (42).

Take $\sigma(t)=1, \varrho(t)=3 / 2$. After standard computation, we get

$$
\begin{aligned}
& \int_{0}^{1} G_{1}(t, s) \sigma(s) d s=\int_{0}^{1} G_{2}(t, s) \sigma(s) d s=\frac{4 t-t^{2}}{6} \\
& \int_{0}^{1} H_{1}(t, s) \sigma(s) d s=\frac{t}{6} \\
& \int_{0}^{1} H_{2}(t, s) \sigma(s) d s=\frac{t}{3}
\end{aligned}
$$

Then

$$
\begin{aligned}
T_{1}(\sigma, \varrho)(t)= & \int_{0}^{1} G_{1}(t, s)(\sigma(s)+\varrho(s)) d s \\
& +\int_{0}^{1} H_{1}(t, s)(2 \sigma(s)+\varrho(s)) d s \\
= & \frac{20 t-5 t^{2}}{12}+\frac{7 t}{12}=\frac{27 t-5 t^{2}}{12}, \\
T_{2}(\sigma, \varrho)(t)= & \int_{0}^{1} G_{2}(t, s)(2 \sigma(s)+\varrho(s)) d s \\
& +\int_{0}^{1} H_{2}(t, s)(\sigma(s)+\varrho(s)) d s \\
= & \frac{28 t-7 t^{2}}{12}+\frac{5 t}{6}=\frac{38 t-7 t^{2}}{12}
\end{aligned}
$$

So it follows from the definition of the norm for linear operator that

$$
\|T\| \geq \frac{\|T(\sigma, \varrho)\|_{E}}{\|(\sigma, \varrho)\|_{E}}=\frac{31}{18}>1 .
$$

Thus Example 5 shows that Theorem 4 provides the same results with weaker conditions.

\section{Data Availability}

The data used to support the findings of this study are included within the article.

\section{Conflicts of Interest}

The authors declare that they have no conflicts of interest.

\section{Acknowledgments}

The project is supported by the National Natural Science Foundation of China (11371221, 11571207), Shandong Natural Science Foundation (ZR2018MA011), and the Tai'shan Scholar Engineering Construction Fund of Shandong Province of China.

\section{References}

[1] M. Pedersen and Z. Lin, "Blow-up analysis for a system of heat equations coupled through a nonlinear boundary condition," Applied Mathematics Letters, vol. 14, no. 2, pp. 171-176, 2001.

[2] A. A. Leung, "A semilinear reaction-diffusion prey-predator system with nonlinear coupled boundary conditions: equilibrium and stability," Indiana University Mathematics Journal, vol. 31, no. 2, pp. 223-241, 1982.

[3] F. Ali Mehmeti and S. Nicaise, "Nonlinear interaction problems," Nonlinear Analysis. Theory, Methods \& Applications. An International Multidisciplinary Journal, vol. 20, no. 1, pp. 27-61, 1993.

[4] N. A. Asif and R. A. Khan, "Positive solutions to singular system with four-point coupled boundary conditions," Journal of Mathematical Analysis and Applications, vol. 386, no. 2, pp. 848-861, 2012.

[5] Y. Cui and J. Sun, "On existence of positive solutions of coupled integral boundary value problems for a nonlinear singular superlinear differential system," Electronic Journal of Qualitative Theory of Differential Equations, vol. 41, pp. 1-13, 2012.

[6] Y. Cui and Y. Zou, "An existence and uniqueness theorem for a second order nonlinear system with coupled integral boundary value conditions," Applied Mathematics and Computation, vol. 256, pp. 438-444, 2015.

[7] Y. Cui, L. Liu, and X. Zhang, "Uniqueness and existence of positive solutions for singular differential systems with coupled integral boundary value problems," Abstract and Applied Analysis, vol. 2013, Article ID 340487, 9 pages, 2013.

[8] Y. Cui and Y. Zou, "Monotone iterative method for differential systems with coupled integral boundary value problems," Boundary Value Problems, vol. 2013, p. 245, 2013.

[9] Y. Cui, "Existence of solutions for coupled integral boundary value problem at resonance," Publicationes Mathematicae, vol. 89, no. 1-2, pp. 73-88, 2016.

[10] G. Infante, F. M. Minhós, and P. Pietramala, "Non-negative solutions of systems of ODEs with coupled boundary conditions," Communications in Nonlinear Science and Numerical Simulation, vol. 17, no. 12, pp. 4952-4960, 2012.

[11] Y. Cui and Y. Zou, "Existence of solutions for second-order integral boundary value problems," Lithuanian Association of Nonlinear Analysts. Nonlinear Analysis: Modelling and Control, vol. 21, no. 6, pp. 828-838, 2016.

[12] J. Henderson, R. Luca, and A. Tudorache, "On a system of fractional differential equations with coupled integral boundary conditions," Fractional Calculus and Applied Analysis, vol. 18, no. 2, pp. 361-386, 2015.

[13] J. Jiang, L. Liu, and Y. Wu, "Symmetric positive solutions to singular system with multi-point coupled boundary conditions," 
Applied Mathematics and Computation, vol. 220, pp. 536-548, 2013.

[14] J. Jiang, L. Liu, and Y. Wu, "Positive solutions to singular fractional differential system with coupled boundary conditions," Communications in Nonlinear Science and Numerical Simulation, vol. 18, no. 11, pp. 3061-3074, 2013.

[15] L. Liu, H. Li, C. Liu, and Y. Wu, "Existence and uniqueness of positive solutions for singular fractional differential systems with coupled integral boundary conditions," The Journal of Nonlinear Science and its Applications, vol. 10, no. 1, pp. 243-262, 2017.

[16] C. $\mathrm{Lu}, \mathrm{C} . \mathrm{Fu}$, and H. Yang, "Time-fractional generalized Boussinesq equation for Rossby solitary waves with dissipation effect in stratified fluid and conservation laws as well as exact solutions," Applied Matheamtics and Computation, vol. 327, pp. 104-116, 2018.

[17] T. Qi, Y. Liu, and Y. Zou, "Existence result for a class of coupled fractional differential systems with integral boundary value conditions," Journal of Nonlinear Sciences and Applications. JNSA, vol. 10, no. 7, pp. 4034-4045, 2017.

[18] Q. Sun and Y. Cui, "Existence results for $(k, n-k)$ conjugate boundary-value problems with integral boundary conditions at resonance with $\operatorname{ker} L=2$," Boundary Value Problems, vol. 1, p. 29, 2017.

[19] C. Yuan, D. Jiang, D. O’Regan, and R. P. Agarwal, "Multiple positive solutions to systems of nonlinear semipositone fractional differential equations with coupled boundary conditions," Electronic Journal of Qualitative Theory of Differential Equations, vol. 13, p. 17, 2012.

[20] Yumei Zou, Lishan Liu, and Yujun Cui, "The Existence of Solutions for Four-Point Coupled Boundary Value Problems of Fractional Differential Equations at Resonance," Abstract and Applied Analysis, vol. 2014, pp. 1-8, 2014.

[21] Y. Zou and G. He, "A fixed point theorem for systems of nonlinear operator equations and applications to $\left(p_{1}, p_{2}\right)$ Laplacian system," Mediterranean Journal of Mathematics, vol. 15, no. 2, Art. 74, 11 pages, 2018.

[22] L. Guo, L. Liu, and Y. Wu, "Uniqueness of iterative positive solutions for the singular fractional differential equations with integral boundary conditions," Boundary Value Problems, vol. 2016, p. 147, 2016.

[23] X. Lin, D. Jiang, and X. Li, "Existence and uniqueness of solutions for singular fourth-order boundary value problems," Journal of Computational and Applied Mathematics, vol. 196, no. 1, pp. 155-161, 2006.

[24] D. Min, L. Liu, and Y. Wu, "Uniqueness of positive solutions for the singular fractional differential equations involving integral boundary value conditions," Boundary Value Problems, vol. 2018, 23 pages, 2018.

[25] X. Zhang, L. Liu, and Y. Wu, "Existence and uniqueness of iterative positive solutions for singular Hammerstein integral equations," Journal of Nonlinear Sciences and Applications. JNSA, vol. 10, no. 7, pp. 3364-3380, 2017.

[26] Z. Bai, "On positive solutions of a nonlocal fractional boundary value problem," Nonlinear Analysis: Theory, Methods \& Applications, vol. 72, no. 2, pp. 916-924, 2010.

[27] Y. Cui, W. Ma, Q. Sun, and X. Su, "New uniqueness results for boundary value problem of fractional differential equation," Nonlinear Analysis: Modelling and Control, pp. 31-39, 2018.

[28] X. Zhang and Q. Zhong, "Uniqueness of solution for higherorder fractional differential equations with conjugate type integral conditions," Fractional Calculus and Applied Analysis, vol. 20, no. 6, pp. 1471-1484, 2017.

[29] Y. Zou and G. He, "On the uniqueness of solutions for a class of fractional differential equations," Applied Mathematics Letters, vol. 74, pp. 68-73, 2017.

[30] Y. Cui, "Uniqueness of solution for boundary value problems for fractional differential equations," Applied Mathematics Letters, vol. 51, pp. 48-54, 2016.

[31] Y. Cui, W. Ma, X. Wang, and X. Su, "Uniqueness theorem of differential system with coupled integral boundary conditions," Electronic Journal of Qualitative Theory of Differential Equations, vol. 9, pp. 1-10, 2018. 


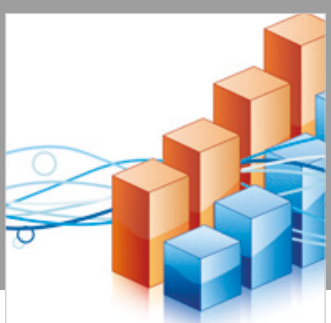

Advances in

Operations Research

\section{-n-m}
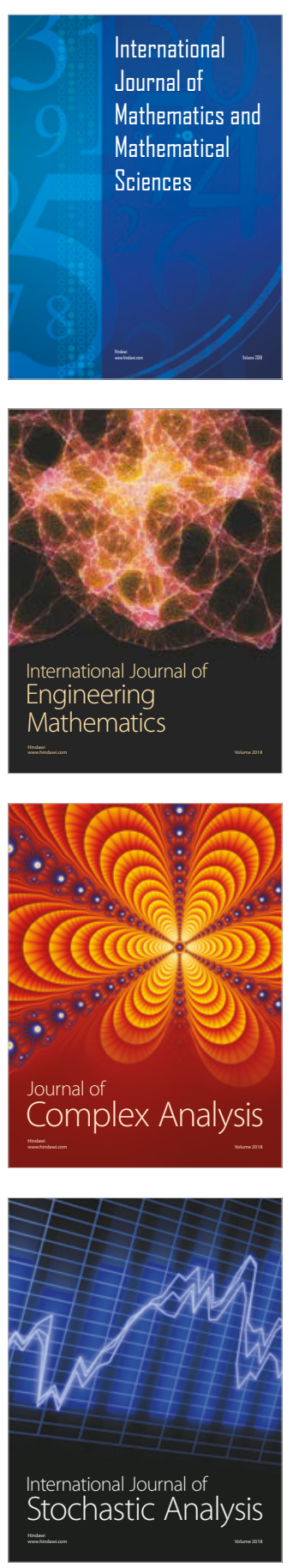
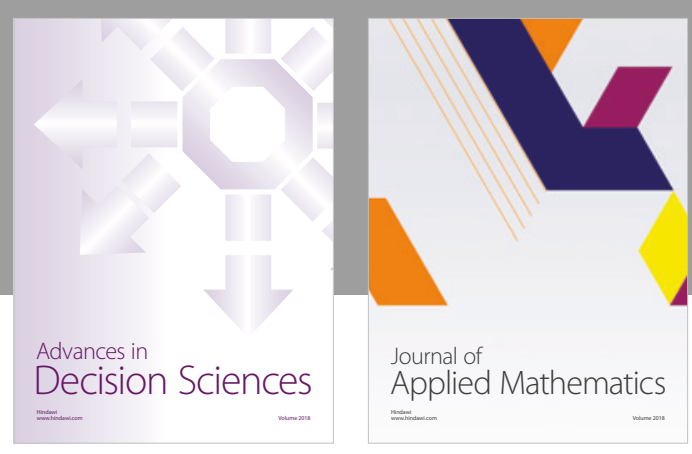

Journal of

Applied Mathematics
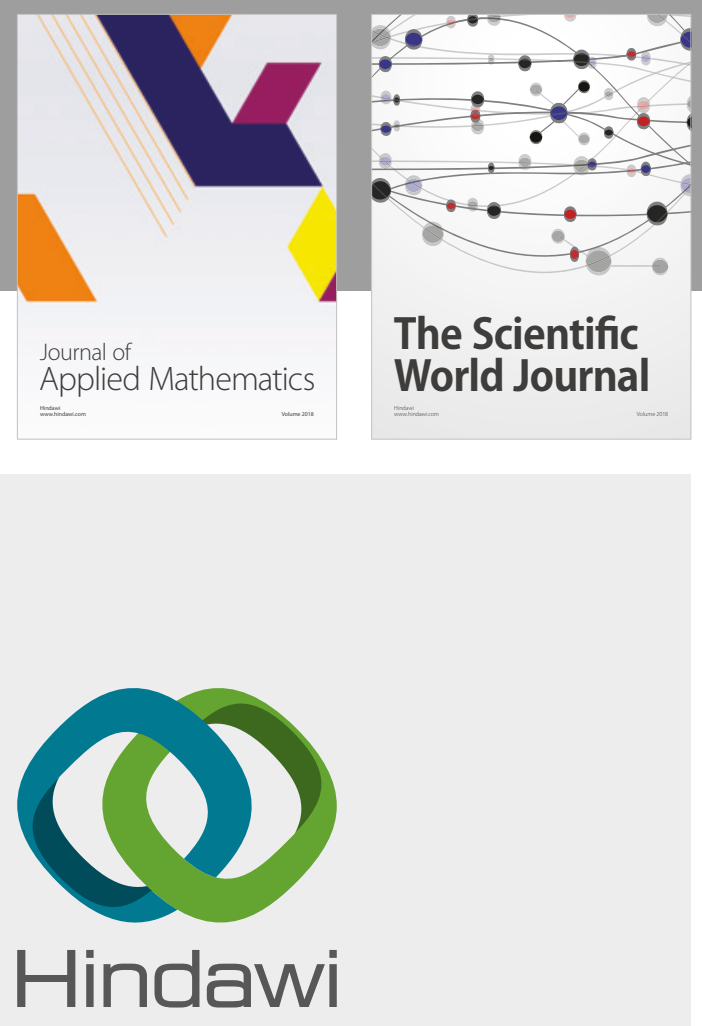

Submit your manuscripts at

www.hindawi.com

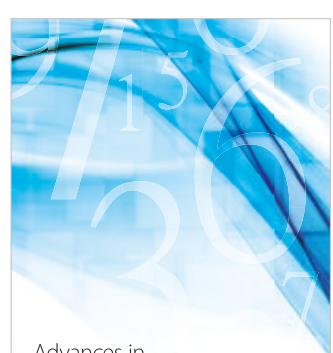

Advances in
Numerical Analysis
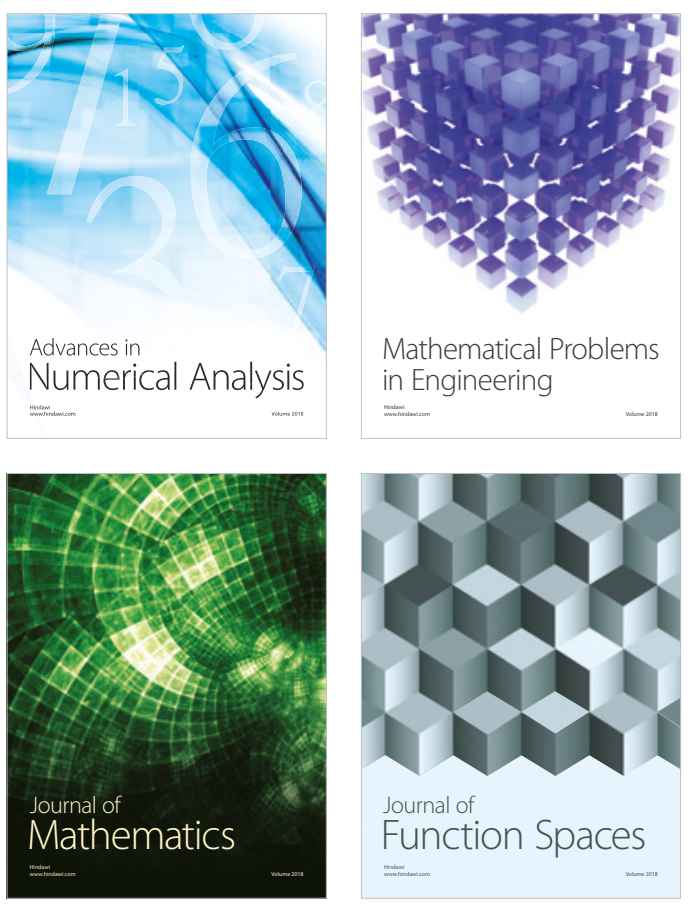

Mathematical Problems in Engineering

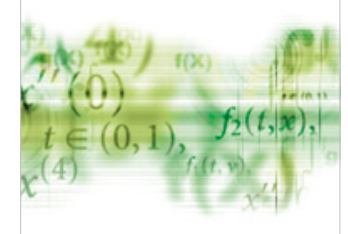

International Journal of

Differential Equations

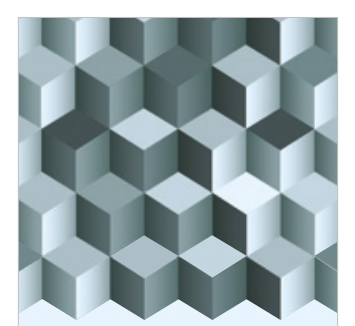

Journal of

Function Spaces

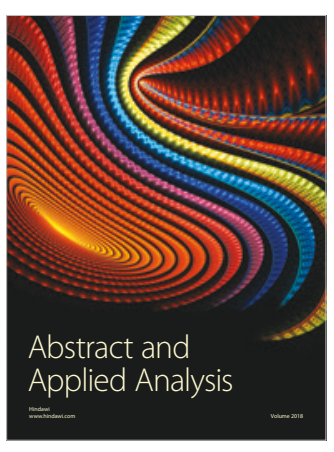

The Scientific

World Journal

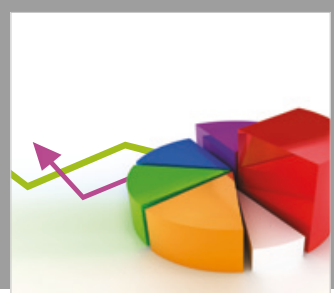

Journal of

Probability and Statistics
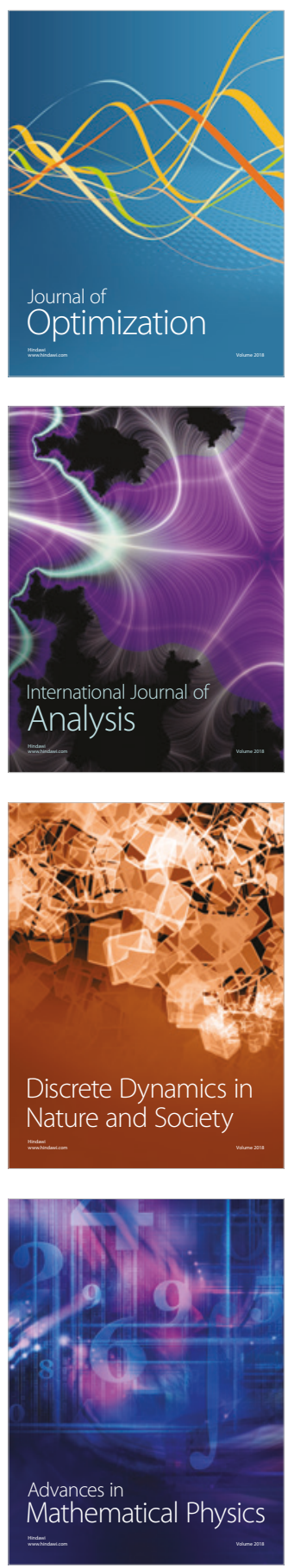